\title{
Improved limits on solar axions and bosonic dark matter from the CDEX-1B experiment using the profile likelihood ratio method
}

Y. Wang, ${ }^{1,2}$ Q. Yue $\odot,{ }^{1, *}$ S. K. Liu, ${ }^{3, \dagger}$ K. J. Kang, ${ }^{1}$ Y. J. Li, ${ }^{1}$ H. P. An,${ }^{1,2}$ J. P. Chang,${ }^{4}$ J. H. Chen, ${ }^{5}$ Y. H. Chen, ${ }^{6}$ J. P. Cheng, ${ }^{1}$ W. H. Dai, ${ }^{1}$ Z. Deng, ${ }^{1}$ X. P. Geng, ${ }^{1}$ H. Gong, ${ }^{1}$ P. Gu, ${ }^{3}$ X. Y. Guo, ${ }^{6}$ H. T. He, ${ }^{3}$ L. He, ${ }^{4}$ S. M. He, ${ }^{6}$ J. W. Hu, ${ }^{1}$ H. X. Huang, ${ }^{7}$ T. C. Huang, ${ }^{8}$ L. P. Jia, ${ }^{1}$ H. B. Li ${ }^{5}$ H. Li, ${ }^{4}$ M.X. Li ${ }^{3}$ J. M. Li, ${ }^{1}$ J. Li ${ }^{1}{ }^{1}$ X. Li, ${ }^{7}$ X. Q. Li ${ }^{9}$ Y. L. Li,${ }^{1}$ B. Liao, ${ }^{10}$ F. K. Lin, ${ }^{5}$ S. T. Lin,${ }^{3}$ Y. D. Liu ${ }^{10}$ Y. Y. Liu, ${ }^{10}$ Z. Z. Liu, ${ }^{1}$ H. Ma, ${ }^{1}$ Q. Y. Nie, ${ }^{1}$ J. H. Ning, ${ }^{6}$ H. Pan, ${ }^{4}$ N. C. Qi,${ }^{6}$ C. K. Qiao, ${ }^{3}$ J. Ren, ${ }^{7}$ X. C. Ruan, ${ }^{7}$ V. Sharma, ${ }^{5,11}$ Z. She, ${ }^{1}$ L. Singh,${ }^{5,11}$ M. K. Singh,${ }^{5,11}$ T. X. Sun, ${ }^{10}$ C. J. Tang, ${ }^{3}$ W. Y. Tang, ${ }^{1}$ Y. Tian, ${ }^{1}$ G. F. Wang, ${ }^{10}$ L. Wang, ${ }^{12}$ Q. Wang, ${ }^{1}$ Z. Wang, ${ }^{3}$ H. T. Wong, ${ }^{5}$ S. Y. Wu, ${ }^{6}$ Y. C. Wu ${ }^{1}$ H. Y. Xing, ${ }^{3}$ Y. Xu, ${ }^{9}$ T. Xue, ${ }^{1}$ Y. L. Yan, ${ }^{3}$ L. T. Yang, ${ }^{1}$ N. Yi, ${ }^{1}$ C. X. Yu, ${ }^{9}$ H. J. Yu, ${ }^{4}$ J. F. Yue, ${ }^{6}$ X. H. Zeng, ${ }^{6}$ M. Zeng, ${ }^{1}$ Z. Zeng, ${ }^{1}$ B. T. Zhang, ${ }^{1}$ F. S. Zhang, ${ }^{10}$ L. Zhang, ${ }^{3}$ Z. Y. Zhang, ${ }^{1}$ M. G. Zhao, ${ }^{9}$ J. F. Zhou, ${ }^{6}$ Z. Y. Zhou, ${ }^{7}$ and J. J. Zhu ${ }^{3}$

(CDEX Collaboration)

\author{
${ }^{1}$ Key Laboratory of Particle and Radiation Imaging (Ministry of Education) and \\ Department of Engineering Physics, Tsinghua University, Beijing 100084 \\ ${ }^{2}$ Department of Physics, Tsinghua University, Beijing 100084 \\ ${ }^{3}$ College of Physics, Sichuan University, Chengdu 610064 \\ ${ }^{4}$ NUCTECH Company, Beijing 100084 \\ ${ }^{5}$ Institute of Physics, Academia Sinica, Taipei 11529 \\ ${ }^{6}$ YaLong River Hydropower Development Company, Chengdu 610051 \\ ${ }^{7}$ Department of Nuclear Physics, China Institute of Atomic Energy, Beijing 102413 \\ ${ }^{8}$ Sino-French Institute of Nuclear and Technology, Sun Yat-sen University, Zhuhai 519082 \\ ${ }^{9}$ School of Physics, Nankai University, Tianjin 300071 \\ ${ }^{10}$ College of Nuclear Science and Technology, Beijing Normal University, Beijing 100875 \\ ${ }^{11}$ Department of Physics, Banaras Hindu University, Varanasi 221005 \\ ${ }^{12}$ Department of Physics, Beijing Normal University, Beijing 100875
}

(Received 11 November 2019; accepted 7 February 2020; published 5 March 2020)

\begin{abstract}
We present the improved constraints on couplings of solar axions and more generic bosonic dark matter particles using $737.1 \mathrm{~kg}$ days of data from the CDEX-1B experiment. The CDEX-1B experiment, located at the China Jinping Underground Laboratory, primarily aims at the direct detection of weakly interacting massive particles using a p-type point-contact germanium detector. We adopt the profile likelihood ratio method for analysis of data in the presence of backgrounds. An energy threshold of $160 \mathrm{eV}$ was achieved, much better than the $475 \mathrm{eV}$ of CDEX-1A with an exposure of $335.6 \mathrm{~kg}$ days. This significantly improves the sensitivity for the bosonic dark matter below $0.8 \mathrm{keV}$ among germanium detectors. Limits are also placed on the coupling $g_{A e}<2.48 \times 10^{-11}$ from Compton, bremsstrahlung, atomic-recombination, and deexcitation channels and $g_{A N}^{\text {eff }} \times g_{A e}<4.14 \times 10^{-17}$ from a ${ }^{57} \mathrm{Fe}$ M1 transition at $90 \%$ confidence level.
\end{abstract}

DOI: 10.1103/PhysRevD.101.052003

\section{INTRODUCTION}

${ }^{*}$ Corresponding author. yueq@mail.tsinghua.edu.cn

${ }^{\dagger}$ Corresponding author. liusk@scu.edu.cn

Published by the American Physical Society under the terms of the Creative Commons Attribution 4.0 International license. Further distribution of this work must maintain attribution to the author(s) and the published article's title, journal citation, and DOI. Funded by SCOAP .
For the charge-parity $(C P)$ problem of strong interactions, the Peccei-Quinn (PQ) mechanism [1] is still the most compelling solution in which a new kind of $U(1)$ symmetry would be spontaneously broken at large energy scale $f_{A}$. After this original solution to the $C P$ conservation in QCD, a new Nambu-Glodstone boson called axion is proposed later by Weinberg [2] and Wilczek [3] through the PQ symmetry. Axions are pseudoscalar particles with properties closely related to those of neutral pions and their 
mass $m_{A}$ is fixed by the scale $f_{A}$ of the PQ symmetry breaking, $m_{A} \approx 6 \mathrm{eV}\left(10^{6} \mathrm{GeV} / f_{A}\right)$. The range of scale $f_{A}$ cannot be restricted by theory, but the order of the electroweak scale has been excluded by experiments. At a higher symmetry-breaking energy scale, "invisible" axion models such as hadronic model KSVZ (Kim-ShifmanVainstein-Zakharov) [4,5] and nonhadronic model DFSZ (Dine-Fischler-Srednicki-Zhitnitskii) [6,7] are still allowed. Another interest in this paper is more general bosonic dark matter (DM) like axionlike particles (ALPs) and vector bosonic DM, which also have couplings to electrons.

Several experiments have reported the corresponding results [8-23] using the mechanism arising from the couplings to electrons,

$$
A(B)+e+Z \rightarrow e+Z,
$$

where $A$ and $B$ represent axion and bosonic DM, respectively. This effect is similar to photoelectric effect just replacing photon with axion or bosonic DM.

The China Dark Matter Experiment (CDEX) is primarily designed to carry out direct detection of low mass weakly interacting massive particles (WIMPs) with p-type pointcontact germanium detectors (PPCGe) at China Jinping Underground Laboratory (CJPL) [24-30]. With a vertical rock overburden of $2.4 \mathrm{~km}$, CJPL provides a measured muon flux of $61.7 \pm 11.7 \mathrm{y}^{-1} \mathrm{~m}^{-2}$ [31]. Besides the WIMPs constraints [32,33], the axion searches results from the CDEX-1A experiment based on the $335.6 \mathrm{~kg}$ days of data have been reported before [18]. Using a PPCGe with fiducial mass of $915 \mathrm{~g}$, a physics threshold of $475 \mathrm{eV}$ [33] was achieved for CDEX-1A. Focused on the lower energy threshold, a new $1 \mathrm{~kg}$ scale PPCGe detector has been designed and named "CDEX-1B" based on the experience from our previous prototype detector used in CDEX-1A.

In this paper, we report the solar axion, ALPs, and vector bosonic DM search results from the CDEX-1B experiment based on the $737.1 \mathrm{~kg}$ days of data, which is the same dataset in the analysis of WIMP search [34], annual modulation [30], and Midgal effects [29]. Also, we describe the statistical model with profile likelihood ratio method applied to these data.

\section{AXION SEARCHES WITH CDEX-1B}

\section{A. CDEX-1B setup and overview}

The CDEX-1B experiment adopts one $939 \mathrm{~g}$ singleelement PPCGe crystal with dead layer of $0.88 \pm 0.12 \mathrm{~mm}$ [35]. Outside of the PPCGe detector is the passive shielding system and the detailed information is described in Ref. [34]. A well-shaped cylindrical $\mathrm{NaI}(\mathrm{Tl})$ crystal surrounding the PPCGe detector is used as the anti-Compton detector. The coincidence events both in germanium and $\mathrm{NaI}(\mathrm{Tl})$ crystals denoted as $\mathrm{AC}^{+}$are discarded to depress the $\gamma$ background.
The schematic diagram of electronics and data acquisition (DAQ) system is shown in Ref. [34]. Four identical energyrelated signals were out of the $p^{+}$point-contact electrode after a pulsed-reset feedback preamplifier. Two of them were distributed into the shaping amplifiers at $6 \mu \mathrm{s}\left(\mathrm{SA}_{6 \mu \mathrm{s}}\right)$ and $12 \mu \mathrm{s}\left(\mathrm{SA}_{12 \mu \mathrm{s}}\right)$ shaping time for low energy region $(0-12 \mathrm{keV})$. The output of $\mathrm{SA}_{6 \mu}$ s provided the system trigger of the DAQ. The other two outputs were fed into timing amplifiers (TA) which provide the accurate time information. One with high gain $\left(\mathrm{TA}_{1}\right)$ is limited to medium energy region ( $0-20 \mathrm{keV})$, and the other one with low gain $\left(\mathrm{TA}_{2}\right)$ for high energy can reach $1.3 \mathrm{MeV}$. The energy resolution of $\mathrm{TA}_{1}$ output is similar to the $\mathrm{SA}_{6 \mu \mathrm{s}, 12 \mu \mathrm{s}}$. As a result, the spectrum below $12 \mathrm{keV}$ is from $\mathrm{SA}_{6} \mu \mathrm{s}$ and above $12 \mathrm{keV}$ is from $\mathrm{TA}_{1}$ in our analysis. The energy resolution $(\sigma)$ from $\mathrm{SA}_{6 \mu}$ at $1.3 \mathrm{keV}$ is about $44 \mathrm{eV}$.

\section{B. Particle sources}

\section{Solar axions}

The Sun is a potential source of axions and in this paper we concentrate on two different mechanisms.

The first important source is the $14.4 \mathrm{keV}$ monochromatic axions from the $\mathrm{M} 1$ transition of the ${ }^{57} \mathrm{Fe}$ in the sun, i.e., ${ }^{57} \mathrm{Fe}^{*} \rightarrow{ }^{57} \mathrm{Fe}+\mathrm{A}$, due to the stability and the large abundance of ${ }^{57} \mathrm{Fe}$ in the sun.

The Lagrangian coupling axions to nucleons is [12]

$$
\mathcal{L}=i \bar{\psi}_{N} \gamma_{5}\left(g_{A N}^{0}+g_{A N}^{3} \tau_{3}\right) \psi_{N} \phi_{A},
$$

where $\psi_{N}$ is the nucleon isospin doublet, $\phi_{A}$ is the axion field, and $\tau_{3}$ is Pauli matrix. $g_{A N}^{0}$ and $g_{A N}^{3}$ are the modeldependent isoscalar and isovector axion-nucleon coupling constants [36,37]. Introducing $g_{A N}^{\text {eff }} \equiv\left(-1.19 g_{A N}^{0}+g_{A N}^{3}\right)$ as the effective nuclear coupling adapted to the case of ${ }^{57} \mathrm{Fe}$, the corresponding axion flux is given by $[12,38]$

$$
\Phi_{14.4}=\left(\frac{\kappa_{A}}{\kappa_{\gamma}}\right)^{3} \times 4.56 \times 10^{23}\left(g_{A N}^{\mathrm{eff}}\right)^{2} \mathrm{~cm}^{-2} \mathrm{~s}^{-1},
$$

where $\kappa_{A}$ and $\kappa_{\gamma}$ are the momenta of the outgoing axion and photon, respectively. Given the axion-nucleon couplings $g_{A N}^{0}$ and $g_{A N}^{3}$ for specific models such as DFSZ and KSVZ, the axion flux can be evaluated.

Other important sources are from the Compton-like scattering (C), axion-bremsstrahlung (B), atomic-recombination $(\mathrm{R})$, and atomic-deexcitation (D) processes. Their corresponding effective Lagrangian is given by [12]

$$
\mathcal{L}=i g_{A e} \bar{\psi}_{e} \gamma_{5} \psi_{e} \phi_{A},
$$

where $g_{A e}$ is the dimensionless axion-electron coupling constant. Its flux depends on the $g_{A e}^{2}$, 


$$
\begin{aligned}
\frac{d \Phi_{C B}}{d E_{A}}= & \frac{d \Phi_{C}}{d E_{A}}+\frac{d \Phi_{B}}{d E_{A}} \\
= & g_{A e}^{2} \times 1.33 \times 10^{33} E_{A}^{2.987} e^{-0.776 E_{A}} \\
& +g_{A e}^{2} \times 2.63 \times 10^{35} E_{A} e^{-0.77 E_{A}} \frac{1}{1+0.667 E_{A}^{1.278}},
\end{aligned}
$$

where the units of fluxes are $\mathrm{cm}^{-2} \mathrm{~s}^{-1} \mathrm{keV}^{-1}$ and axion energy $E_{A}$ is in unit of keV. For the atomic-recombination and atomic-deexcitation process, the tabulated spectrum in Ref. [39] is used. As discussed in Ref. [39], the flux is valid for relativistic axion; hence, we consider only the axion mass below $1 \mathrm{keV}$.

The axion-electron coupling is depended on models. In the DFSZ model, the coupling is proportional to $\cos ^{2} \beta$, where $\tan \beta$ is the ratio of the two Higgs vacuum expectation values. In the KSVZ model, it depends on $E / N$, the ratio of electromagnetic to color anomalies. $E / N=0$ and $\cos ^{2} \beta=1$ are used in this analysis [12].

\section{Bosonic dark matter}

The main cosmological interest in bosonic particles such as ALPs and vector bosonic DM arises from their possible role as the dominant component of dark matter, the nature of which is still unknown. The absorption via ionization or excitation of an electron in target atom makes bosonic DM experimentally interesting and PPCGe detectors have advantages to study bosonic DM due to their excellent energy resolution, sub-keV threshold, and low radioactivity background.

Assuming that these bosonic particles constitute all of the galactic dark matter, we get the total average flux of dark matter axions on the Earth,

$$
\begin{aligned}
\Phi_{\mathrm{DM}} & =\rho_{\mathrm{DM}} \cdot v_{A} / m_{A} \\
& =9.0 \times 10^{15} \times \beta \cdot\left(\frac{\mathrm{keV}}{m_{A}}\right) \mathrm{cm}^{-2} \mathrm{~s}^{-1},
\end{aligned}
$$

where $\rho_{\mathrm{DM}} \sim 0.3 \mathrm{GeV} / \mathrm{cm}^{3}$ is the dark matter halo density [40], $m_{A}$ is the axion mass, $v_{A}$ is the mean axion velocity distribution with respect to the Earth, and $\beta$ is the ratio of the axion velocity to the speed of light for cold dark matter. This flux is independent of any axion coupling.

\section{Particle interactions in CDEX-1B}

The axion detection channel studied in this paper is the axioelectric effect illustrated in Eq. (1). The axioelectric cross section as described in Refs. [41-43] is given by

$$
\sigma_{A e}\left(m_{A}\right)=\sigma_{p e}\left(m_{A}\right) \frac{g_{A e}^{2}}{\beta} \frac{3 m_{A}^{2}}{16 \pi \alpha m_{e}^{2}}\left(1-\frac{\beta^{\frac{2}{3}}}{3}\right)
$$

where $\sigma_{p e}\left(m_{A}\right)$ is the photoelectric cross section for germanium in the unit of barns/atom, $m_{A}$ is the mass of

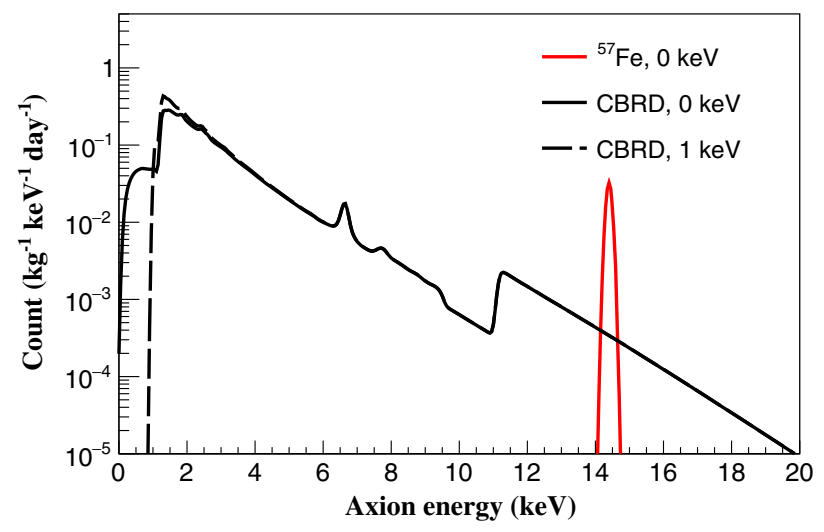

FIG. 1. The expected axion event rates of CBRD process at the mass of 0 and $1 \mathrm{keV}$, and ${ }^{57} \mathrm{Fe} 14.4 \mathrm{keV}$ axion at the mass of $0 \mathrm{keV}$. Here the axion couplings are $g_{A e}=2 \times 10^{-11}$ and $g_{A N}^{\mathrm{eff}} \times g_{A e}=2 \times 10^{-17}$.

axion, $\alpha$ is the fine structure constant, $m_{e}$ is the electron mass, and $\beta$ is the ratio of the axion velocity to the speed of light. The expected axion event rates of CBRD process and ${ }^{57} \mathrm{Fe}$ under the consideration of energy resolution are displayed in Fig. 1.

In the situation of ALPs in cold dark matter model $\left(\beta \approx 10^{-3}\right)$, the coupling to electrons is the same as in the case of solar axions. For the vector bosonic DM, the absorption cross section $\sigma_{\text {abs }}$ can be written as

$$
\sigma_{\mathrm{abs}}\left(m_{v}\right)=\sigma_{p e}\left(m_{v}\right) \frac{\alpha^{\prime}}{\alpha}
$$

where $m_{v}$ is the mass of the vector bosonic DM, $\alpha$ and $\alpha^{\prime}$ are the fine structure constant and its vector boson equivalent, respectively.

Using the parameter mentioned above, the interaction rate in the direct detection experiment can be written as

$$
R=1.2 \times 10^{43} A^{-1} g_{A e}^{2} m_{A} \sigma_{p e}\left(m_{A}\right)
$$

for ALPs and

$$
R=4 \times 10^{47} A^{-1} \frac{\alpha^{\prime}}{\alpha} m_{v}^{-1} \sigma_{p e}\left(m_{v}\right)
$$

for vector bosonic DM, where $A$ is mass number of germanium. The expected rates of these two kinds of particles are shown in Fig. 2.

\section{DATA ANALYSIS}

\section{A. Data selection}

As discussed in earlier analysis [34], the background spectrum is derived by the following steps:

(1) Stability check, removing the time periods of calibration or other testing experiments. 


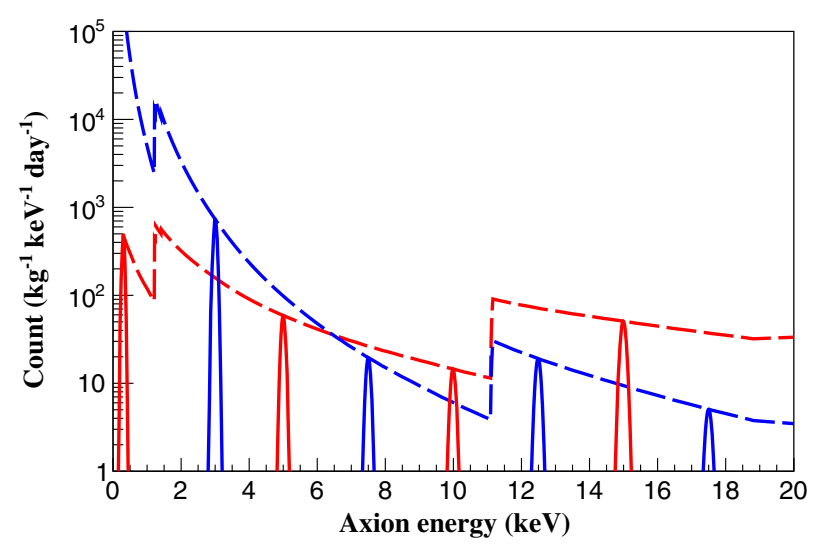

FIG. 2. The expected event rate of ALPs (red solid line) and vector bosonic DM (blue solid line) at different masses. The red dashed line is the maximum event rate of ALPs Gaussian distributions versus their masses, while the blue dashed line is corresponding to vector bosonic DM. The couplings used here are $g_{A e}=2 \times 10^{-11}$ and $\alpha^{\prime} / \alpha=5 \times 10^{-25}$. The widths of these peaks are determined by the energy resolution.

(2) Anti-Compton (AC) veto, discarding the events in coincidence with the $\mathrm{AC}$ detector and retaining the anticoincidence events.

(3) Basic cuts, removing the electronic noise through getting rid of the abnormal pulses and spurious signals.

(4) Bulk and surface event selection, rejecting the surface events by pulse shape analysis using their characteristic slower rise time.

Depicted in Fig. 3 are the trigger efficiency as well as the selection efficiency with energy including those from the selection of physics versus electronic noise events, AC vetos, and DAQ dead time. The trigger efficiency is derived from the calibration sources in coincidence with $\mathrm{AC}$ detector [34]. The selection efficiencies are derived by events due to random triggers, the $\mathrm{AC}$ tagged events from calibration sources, and in situ background. An improved

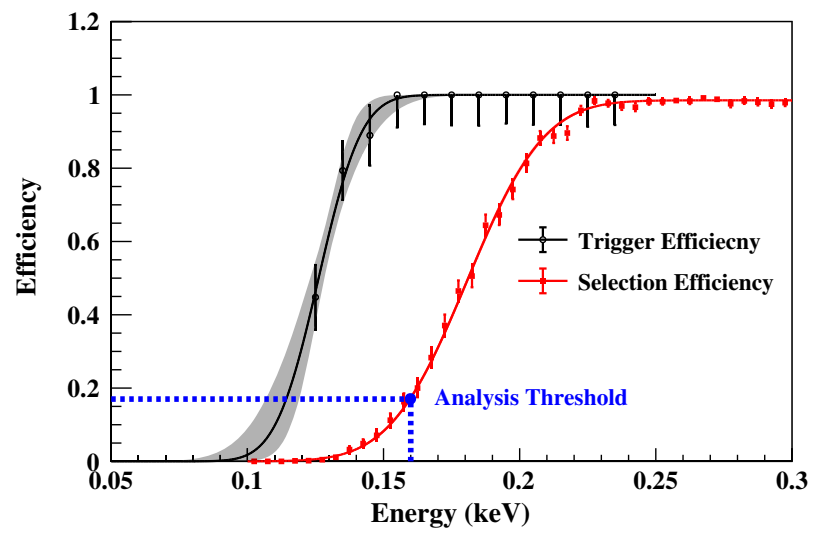

FIG. 3. The trigger efficiency and selection efficiency derived from source events are depicted, respectively, and fitted with error functions. The shadow parts represent the $1 \sigma$ error bands. ratio method, which is based on the bulk/surface rise-time distribution probability density functions (PDFs), is developed to reject the surface events [44]. This method has been proved correctly above $160 \mathrm{eV}$. So, in this analysis, $160 \mathrm{eV}$ is selected as the physics analysis threshold, at which the combined efficiency $\left(\varepsilon_{\text {eff }}\right)$, including trigger and selection, is $17 \%$.

\section{B. Background and understanding}

With an exposure of $737.1 \mathrm{~kg}$ days, the bulk spectrum from $160 \mathrm{eV}$ up to $20 \mathrm{keV}$ after data selection and efficiency correction is displayed in Fig. 4(a). The background consists of several $\mathrm{K}$-shell $\mathrm{x}$ rays and their corresponding $\mathrm{L}$-shell $\mathrm{x}$ rays from the cosmogenic isotopes and $\mathrm{a}$ continuous background with a smooth, slightly increasing profile as the energy decreases [34]. Considering the low muon flux mentioned above, the contribution from muons can be neglected. The continuous background below $20 \mathrm{keV}$ is expected to probably originate from the ${ }^{238} \mathrm{U}$, ${ }^{232} \mathrm{Th}$, and ${ }^{40} \mathrm{~K}$ in the materials in the vicinity of the PPCGe detector, radon gas penetrating through shielding, and cosmogenic ${ }^{3} \mathrm{H}$ in the crystal. A detailed modeling of the continuous background is beyond this work and will be studied in our future work.

However, axion analysis is not sensitive to the accurate background assumption because the signatures of axion are significantly different from the continuous background. As can be seen from Fig. 2, the signal signatures of ${ }^{57} \mathrm{Fe}$ and bosonic DM are monochromatic and of Gaussian

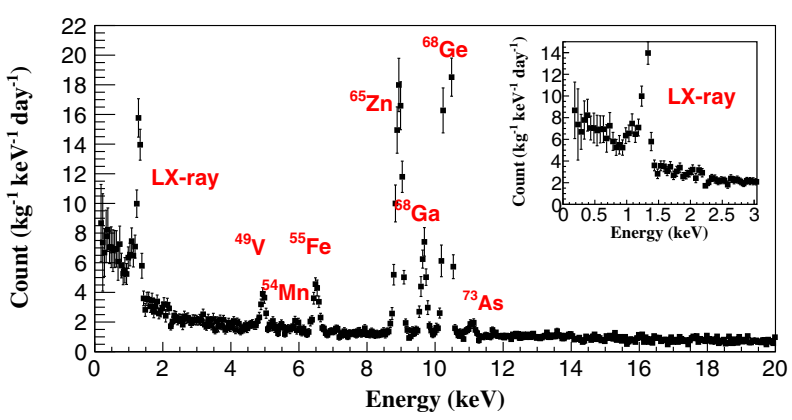

(a)

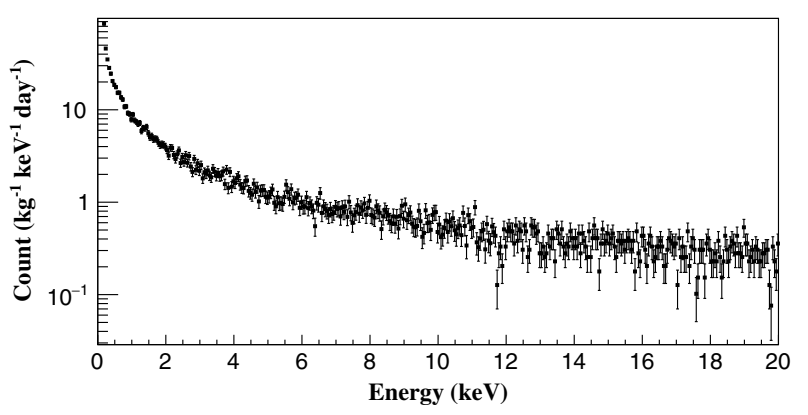

(b)

FIG. 4. (a) The corrected bulk spectrum from $160 \mathrm{eV}$ to $20 \mathrm{keV}$. (b) The corrected surface spectrum from $160 \mathrm{eV}$ up to $20 \mathrm{keV}$. 
distribution with widths determined by the energy resolution. As to the continuous CBRD solar axion, a sawtoothlike profile arises between 0.9 and $1.6 \mathrm{keV}$ considering the axion mass below $1 \mathrm{keV}$. So, in the following fitting procedure, the background model can be described by a continuous background plus the peaks from K/L-shell $\mathrm{x}$ rays. Benefiting from the low threshold and excellent energy resolution of CDEX-1B, the L-shell x-ray peaks at low energy region can be clearly distinguished. Therefore, in the background model, the amplitude of the K-shell x-ray peaks and the corresponding L-shell x-ray peaks are limited by each other using the K/L-shell x-ray ratios mentioned in Refs. [45,46]. In the ultralow energy region around the threshold, M-shell $\mathrm{x}$ rays are also taken into consideration in the background model.

The corrected surface spectrum derived from ratio method is depicted in Fig. 4(b). Note that, as will be clear in next section, the likelihood analysis makes use of both the bulk and surface data.

\section{Profile likelihood analysis}

A profile likelihood analysis, as described in Ref. [47], is adopted to derive the constraints and the test statistics is

$$
q_{\mu}= \begin{cases}-2 \ln \left(\frac{L(\mu, \hat{\hat{\theta}})}{L(\hat{\mu}, \hat{\theta})}\right) & \mu \geq \hat{\mu} \\ 0 & \mu<\hat{\mu},\end{cases}
$$

where $L$ is the likelihood function. Quantity $\mu$ is a parameter corresponding to the strength of signals and $\theta$ denotes all of the nuisance parameters. The quantity $\hat{\hat{\theta}}$ denotes the value of $\theta$ that maximizes $L$ for the specified $\mu$, while the denominator is the maximized likelihood function, i.e., $\mu$ and $\theta$ are their maximum-likelihood estimators. To obtain the $90 \%$ C.L. bounds on the signal strengths $\mu$, the asymptotic formulas are used to calculate the probability distribution functions (PDFs), i.e.,

$$
\begin{aligned}
f\left(q_{\mu} \mid \mu^{\prime}\right)= & \Phi\left(\frac{\mu^{\prime}-\mu}{\sigma}\right) \delta\left(q_{\mu}\right) \\
& +\frac{1}{2} \frac{1}{\sqrt{2 \pi}} \frac{1}{\sqrt{q_{\mu}}} \exp \left[\frac{1}{2}-\left(\sqrt{q_{\mu}}-\frac{\mu-\mu^{\prime}}{\sigma}\right)^{2}\right],
\end{aligned}
$$

where $f\left(q_{\mu} \mid \mu^{\prime}\right)$ is the PDF of the test statistic $q_{\mu}$ under the signal strength hypothesis $\mu^{\prime}$, while $\sigma$ is the corresponding standard deviation [47]. Since downward fluctuations of background might lead to much stringent exclusion results, we used the $\mathrm{CL}_{s}$ method [48] to get rid of this effect. The 90\% up limits $\mu_{\text {up }}$ are defined as

$$
\frac{1-F\left(q_{\mu} \mid \mu\right)_{\text {up }}}{1-F\left(q_{\mu} \mid 0\right)_{\text {up }}}=10 \%,
$$

where $F$ is the cumulative distribution function of the test statistic.

\section{Likelihood function}

The specific full likelihood function $\mathcal{L}$ we used in this analysis is written as a product of three terms,

$$
\begin{aligned}
\mathcal{L}= & \mathcal{L}_{1}\left(\nu_{A}, \nu_{b}, \nu_{s}, g_{b}, g_{s}, \varepsilon_{\text {eff }} ; m_{A}\right) \times \mathcal{L}_{2}\left(\varepsilon_{\text {eff }}(E)\right) \\
& \times \mathcal{L}_{3}\left(t_{b}, t_{s}\right),
\end{aligned}
$$

the parameter of interest becomes the number of fitted axion event numbers denoted as $\nu_{A}$ which is related to the axion-electron coupling strength $g_{A e}$, whereas $\nu_{b}, \nu_{s}, g_{b}, g_{s}$, $\varepsilon_{\text {eff }}$ are considered as the main nuisance parameters.

$$
\begin{aligned}
\mathcal{L}_{1}= & \prod_{j=1}^{N_{\tau}} \prod_{i=1}^{N_{E}} \operatorname{Poisson}\left[n_{i j} \mid\left(g_{b}\left(\tau_{j} ; E_{i} ; t_{b}\right) \cdot \varepsilon_{\mathrm{eff}}\left(E_{i}\right) \cdot N_{i, \text { bulk }}\right.\right. \\
& \left.\left.+g_{s}\left(\tau_{j} ; E_{i} ; t_{s}\right) \cdot \varepsilon_{\mathrm{eff}}\left(E_{i}\right) \cdot N_{i, \text { surf }}\right)\right]
\end{aligned}
$$

describes the measurement of the detector. Here we projected all the data into the energy versus rise-time two-dimensional (2D) grids, as depicted in Fig. 5(a). The $n_{i j}$ is the measured event number both in the energy spectrum bin $E_{i}$ and the rise-time spectrum bin $\tau_{j}$. $g_{b}\left(\tau ; E_{i} ; t_{b}\right)$ and $g_{s}\left(\tau ; E_{i} ; t_{s}\right)$ are the distributions of rise time at the condition of a certain energy bin $i$ from bulk event and surface event, respectively, i.e., $g_{k}\left(\tau_{j} ; E_{i}\right)=$ $\mu_{k}\left(\tau_{j} ; E_{i}\right)+t_{k} \cdot \sigma_{k, i j}, k=$ bulk or surface. Normalized PDFs $\mu_{k}\left(\tau_{j} ; E_{i}\right)$ are the best-fit values derived from ratio method in the rise-time distribution shown in Fig. 5(b), as well as their corresponding errors $\sigma_{k, i j}$ including statistical and systematic uncertainties which have already been derived in Refs. [34,44]. $\varepsilon_{\text {eff }}$ described by $\vec{e}$ refers to the combined efficiencies mentioned in Sec. III. A,

$$
\begin{aligned}
\varepsilon_{\mathrm{eff}}= & \left\{\frac{1}{2} \times\left[1+\operatorname{Erf}\left(\frac{E-e_{1}}{\sqrt{2} e_{2}}\right)\right]\right\}_{\text {trigger }} \\
& \times\left\{\frac{e_{3}}{2} \times\left[1+\operatorname{Erf}\left(\frac{E-e_{4}}{\sqrt{2} e_{5}}\right)\right]\right\}_{\text {selection }} .
\end{aligned}
$$

$N_{i, \text { bulk }}$ and $N_{i, \text { surf }}$ are the expected numbers of bulk events and surface events at the certain energy bin $i$, respectively, which are determined by the fitting results,

$$
\begin{aligned}
N_{i, \text { bulk }} & =\nu_{b} \cdot f_{b}\left(E_{i}\right)+\nu_{A} \cdot f_{A}\left(E_{i}\right) \\
N_{i, \text { surf }} & =\nu_{s} \cdot f_{s}\left(E_{i}\right) .
\end{aligned}
$$

$f_{b}, f_{A}$, and $f_{s}$ represent the PDFs of the background, the axion signal, and the surface events, respectively. Each of them is normalized to unity over the energy range of the fit. $f_{A}$ describes the axion events as shown in Figs. 1 and 2. 


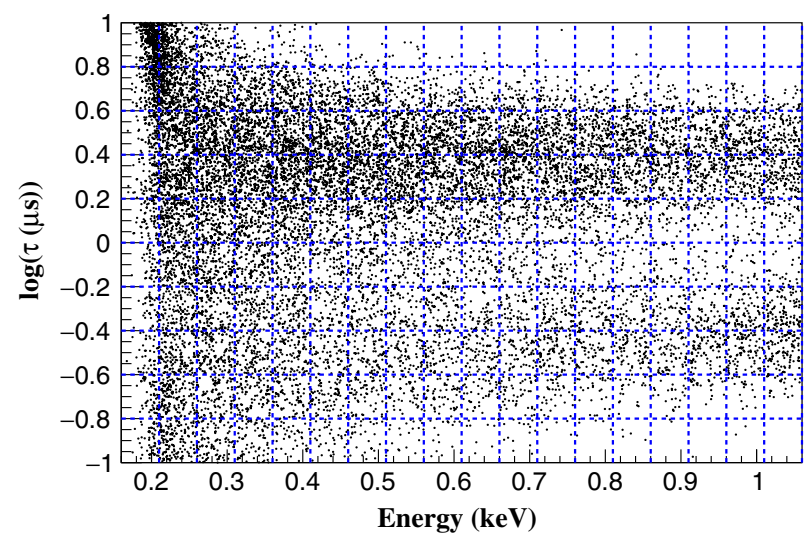

(a)

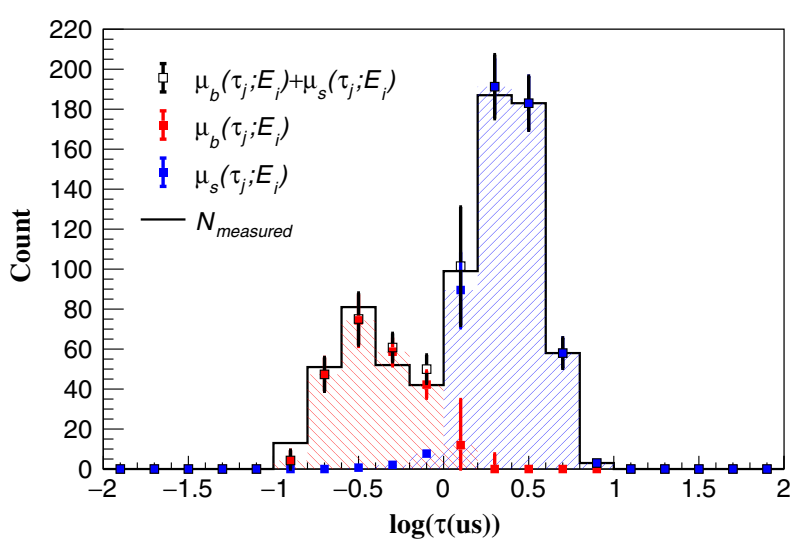

(b)

FIG. 5. (a) The event distribution in the rise time versus energy and the blue dashed grid displayed here shows the method of binning. (b) The distribution of rise time in energy bin 0.66$0.71 \mathrm{keV}$. The $\mu_{b}$ and $\mu_{s}$ are the normalized PDFs of bulk and surface events in this energy bin derived from the output of ratio method and properly scaled to be compared with the measured numbers.

The background $f_{b}$ consists of K-shell x-ray peaks from the cosmogenic nuclides and their corresponding L-shell $\mathrm{x}$-rays and a continuous component with a smooth, slightly increasing profile as the energy decreases. The surface event $f_{s}$ is derived from fitting the surface spectrum with a smooth curve. The systematic uncertainties of the PDF selection of $f_{s}$ are negligible by comparing bin by bin PDFs from the $f_{s}$ spectrum. The number of surface events derived from the ratio method is used as $\nu_{s}$ and fixed in the likelihood fit. The results are consistent with the situation in which $\nu_{s}$ is free, but more conservative below $400 \mathrm{eV}$ in the bosonic DM fit. While $\nu_{b}$ and $\nu_{A}$ fitted as free parameters are the numbers of background events and axion events, respectively.

\section{Constraints and systematic uncertainties}

$\mathcal{L}_{2}$ is a constraint term which encodes prior constraints on the combined efficiencies $\varepsilon_{\text {eff }}$,

$$
\begin{aligned}
\mathcal{L}_{2}= & \exp \left[-\frac{1}{2} \sum_{i, j=1}^{2}\left(e_{i}-\mu_{e i}\right) \boldsymbol{V}_{i j}^{-1}\left(e_{j}-\mu_{e j}\right)\right] \\
& \times \exp \left[-\frac{1}{2} \sum_{i, j=3}^{5}\left(e_{i}-\mu_{e i}\right) \boldsymbol{V}_{i j}^{-1}\left(e_{j}-\mu_{e j}\right)\right] .
\end{aligned}
$$

The five parameters $\vec{e}$ used in two error functions to describe the trigger efficiency and selection efficiency included in $\varepsilon_{\text {eff }}$ are constrained by $\mathcal{L}_{2}$, with $2 \mathrm{D}$ and $3 \mathrm{D}$ Gaussians, respectively. Both centers of the Gaussians are derived by the best-fit values of parameters denoted as $\overrightarrow{\mu_{e}}$ depicted in Fig. 3, and their shapes are determined by the covariance matrix $\boldsymbol{V}$ between the best-fit values.

According to the evaluation in the previous work [34], one of the dominated uncertainties at the energy range below $1 \mathrm{keV}$, including statistical and systematic errors, originate from the bulk surface event selection, i.e., the nuisance parameters $g_{k}\left(\tau_{j} ; E_{i}\right)$, in likelihood function $\mathcal{L}_{1}$. In order to take these uncertainties into consideration, $\mathcal{L}_{3}$ term is introduced,

$$
\mathcal{L}_{3}=e^{-t_{b}^{2} / 2} \times e^{-t_{s}^{2} / 2},
$$

which has been parametrized with two parameters $t_{b}, t_{s}$. The likelihood function is defined to be a product of two normally Gaussian distributions, corresponding to where $t= \pm 1$ corresponds to a $\pm 1 \sigma$ deviation in $g_{k}\left(\tau_{j} ; E_{i}\right)$.

The uncertainties of the background assumption $f_{b}$ are evaluated by using different continuous component in the background assumption between different combinations of exponential, polynominal, and flat functions for the fit below $12 \mathrm{keV}$. For the energy range around $14.4 \mathrm{keV}$, background assumptions are varied between polynomial, flat, and exponential function. The variation of background models causes the change of constraints less than $8 \%$ for CBRD axion, less than $16 \%$ for bosonic DM, and less than $8 \%$ for ${ }^{57} \mathrm{Fe}$ solar axion. As for the uncertainties of resolution, varying the energy resolution by $\pm 10 \%$, the changes of results are less than $17 \%$ for ${ }^{57} \mathrm{Fe}$ solar axion, less than $13 \%$ for bosonic DM, and negligible for CBRD axion.

\section{AXION SENSITIVITY ANALYSIS AND RESULTS}

\section{A. $14.4 \mathrm{keV}$ solar axion}

The signal of solar axions produced in the ${ }^{57} \mathrm{Fe}$ magnetic transition on the spectrum is a monochromatic Gaussian peak around $14.4 \mathrm{keV}$ with width determined by resolution, which is about $84 \mathrm{eV}(\sigma)$ under this situation. The fitting range is limited to $14.06-14.76 \mathrm{keV}$, about $\pm 4 \sigma$, and a polynomial function is used to described the background in this range. The 90\% C.L result is shown in Fig. 6, and the rate of this kind of axion was found to be less than 


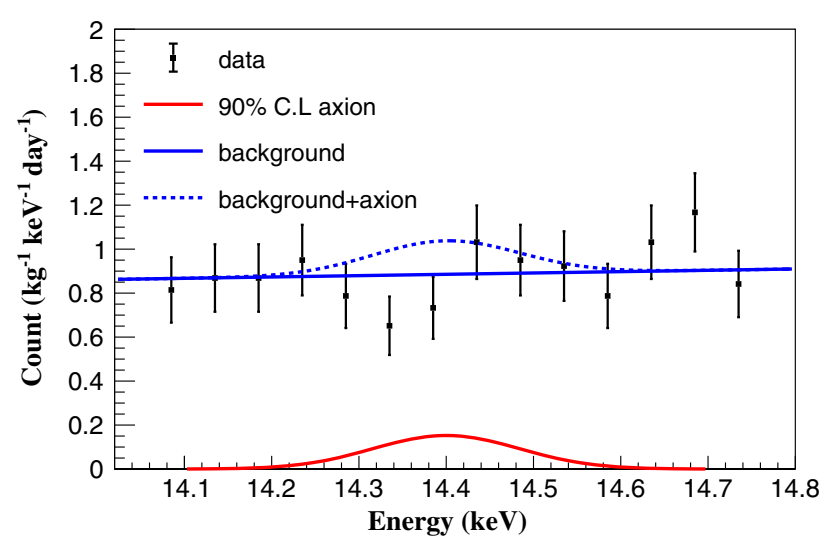

FIG. 6. The bulk data (black data points) and the background assumption (solid blue line), as well as the $90 \%$ C.L ${ }^{57} \mathrm{Fe}$ result (solid red line). The dashed blue line represents the background $+90 \%$ C.L signal.

0.029 counts $\cdot \mathrm{kg}^{-1} \cdot \mathrm{day}^{-1}$. For a low-mass axion at $0 \mathrm{keV}$, this result translates to a $90 \%$ C.L. constraint on the coupling,

$$
g_{A N}^{\text {eff }} \times g_{A e}<4.14 \times 10^{-17} .
$$

Scanning the axion mass from 0 to $14.4 \mathrm{keV}$, we obtained the model-independent limit of $g_{A N}^{\text {eff }} \times g_{A e}$ shown in Fig. 7 .

Within the framework of a specific axion model, KSVZ or DFSZ, the limits on the couplings $g_{A e}$ can constrain axion mass $m_{A}$ directly. Using the assumption of parameters mentioned in Sec. II B, CDEX-1B excludes the mass range $7.3 \mathrm{eV} / \mathrm{c}^{2}<m_{A}<14.4 \mathrm{keV} / \mathrm{c}^{2}$ for DFSZ axions and $141.2 \mathrm{eV} / \mathrm{c}^{2}<m_{A}<14.4 \mathrm{keV} / \mathrm{c}^{2}$ for KSVZ axions.

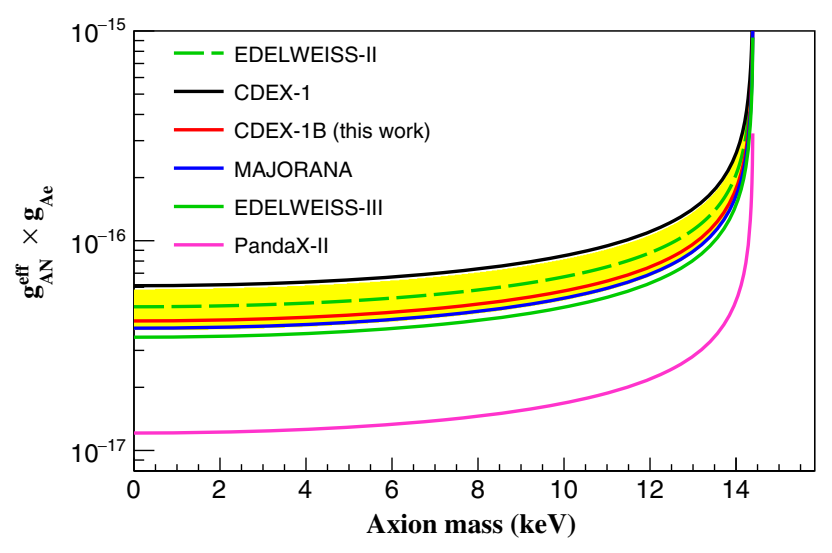

FIG. 7. 90\% C.L. upper limit for the model-independent coupling of $g_{A N}^{\text {eff }} \times g_{A e}$ of ${ }^{57} \mathrm{Fe} 14.4 \mathrm{keV}$ solar axion (solid red line), compared with CDEX-1A [18], EDELWEISS-II [12], EDELWEISS-III [20], Majorana Demonstrator [14], and PandaX-II [16]. The yellow band represents the $1 \sigma$ expected sensitivity.

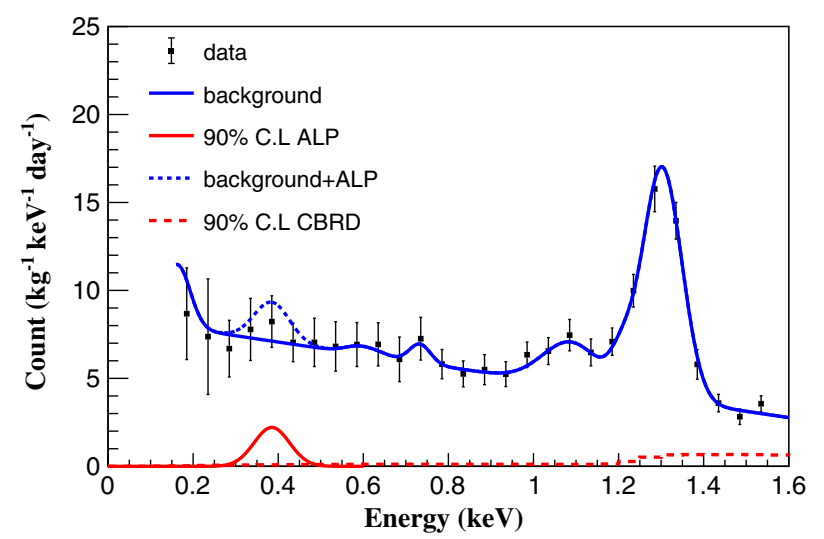

FIG. 8. The bulk data (black data points) and the background assumption (solid blue line) below $1.6 \mathrm{keV}$, as well as the $90 \%$ C.L ALPs result (solid red line) at mass of $385 \mathrm{eV}$ and the 90\% C.L CBRD result (dashed red line). The dashed blue line is the background $+90 \%$ C.L ALP signal.

\section{B. CBRD}

For CBRD solar axions, the fitting range is from 0.8 to $2.0 \mathrm{keV}$, and there is a sawtoothlike profile arising in this energy range which is different from the continuous background. Using the analysis procedure mentioned above, we get the constraints on $g_{A e}$,

$$
g_{A e}<2.48 \times 10^{-11} \text {. }
$$

Figure 8 depicts the fitting results of $90 \%$ C.L. This result, together with other experimental bounds, is displayed in Fig. 9. This result excludes the axion masses $m_{A}>0.9 \mathrm{eV} / \mathrm{c}^{2}$ in the DFSZ model or $m_{A}>257.3 \mathrm{eV} / \mathrm{c}^{2}$ in the KFSZ model, which is better than the result of CDEX-1A.

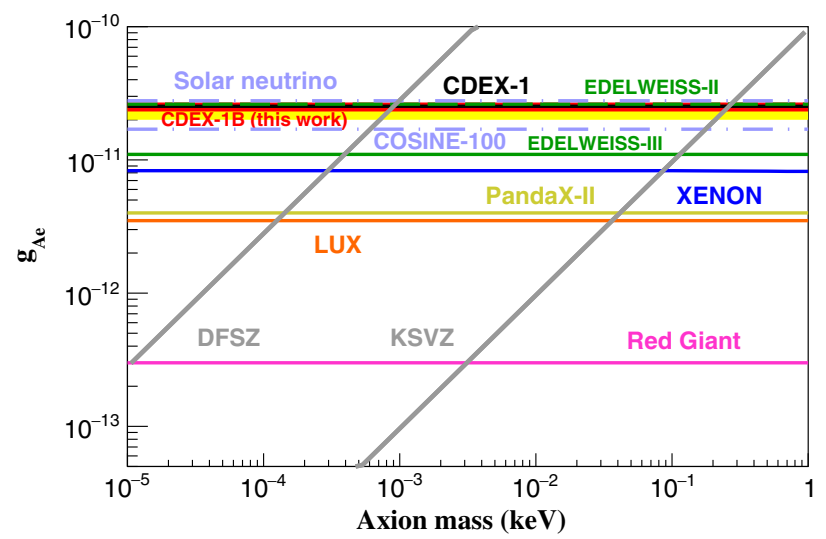

FIG. 9. The CDEX-1B 90\% C.L. on CBRD solar axions (solid red line), together with astrophysical bounds [8,11] and other direct search experiments $[12,15-17,20,23]$. The yellow band represent the $1 \sigma$ expected sensitivity. 


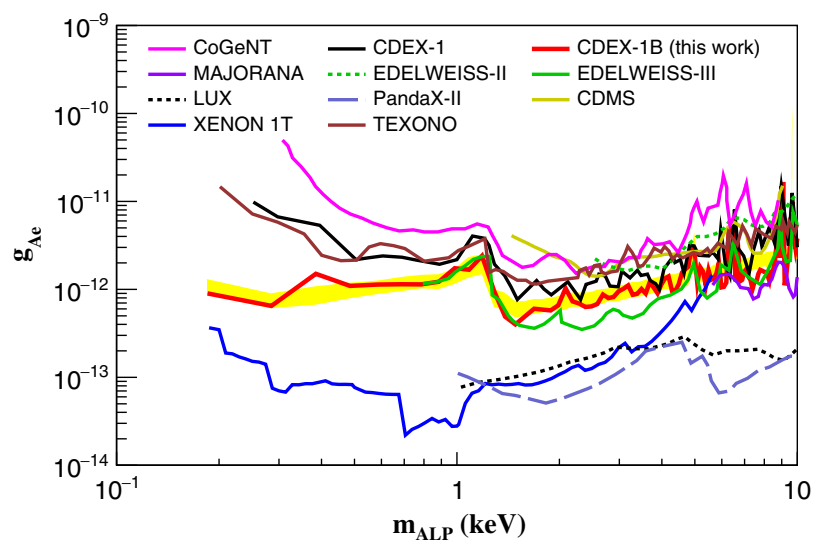

FIG. 10. The CDEX-1B 90\% C.L. upper limit on coupling of ALPs as a function of $m_{\mathrm{ALP}}$, together with the constraints set by CDEX-1A [18] and other experiments [9,10,12,14-16,20-22]. The yellow band represents the $1 \sigma$ expected sensitivity.

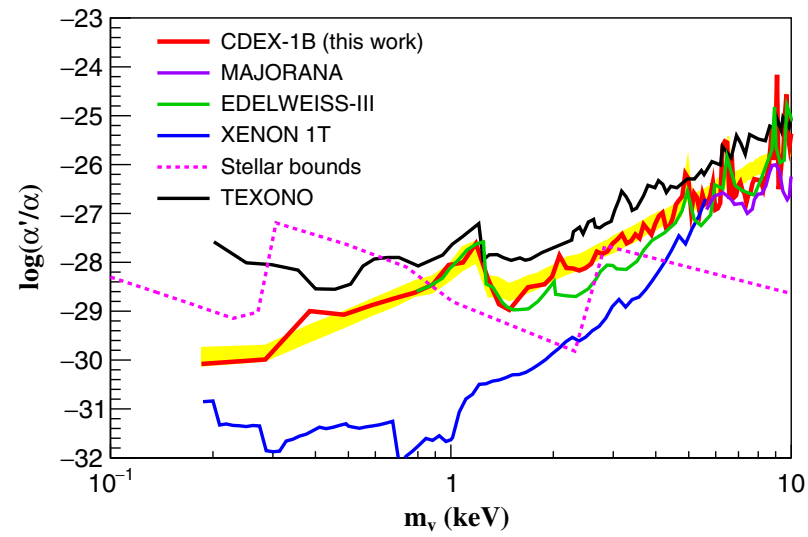

FIG. 11. The $90 \%$ C.L. upper limit on the coupling of vector bosonic DM from CDEX-1B together with the result of EDELWEISS-III [20], Majorana Demonstrator [14], XENON 1T [22], TEXONO [21], and astrophysical bounds from [13]. The yellow band represents the $1 \sigma$ expected sensitivity.

\section{Bosonic dark matter}

For bosonic dark matter, the fitting range is from 0.16 to $11.66 \mathrm{keV}$, and Fig. 8 displays the fitting results at the mass of $385 \mathrm{eV}$ as well as the background model below $1.6 \mathrm{keV}$. Because of the monochromatic signal, better energy resolution and larger exposure, the CDEX-1B gives us much better results of bosonic DM comparing with CDEX1A. The $90 \%$ C.L. limits on $g_{A e}$ of ALPs and $\alpha^{\prime} / \alpha$ of vector bosonic DM are displayed in Figs. 10 and 11, respectively. Due to the lower energy threshold, we can extend the first point of exclusion line down to $185 \mathrm{eV}$.

\section{SUMMARY}

Tighter constraints on the couplings of solar axions and bosonic DM are obtained from CDEX-1B data with an exposure of $737.1 \mathrm{~kg}$ days. Competitive results at the mass of sub-keV of ALPs and vector bosonic DM have been achieved by the help of lower energy threshold and excellent energy resolution measured by the germanium detectors.

These results demonstrate that the profile likelihood ratio method successfully derived the upper limits for our CDEX-1B data in the presence of backgrounds based on the bulk/surface rise-time distribution PDFs. This statistical model takes the main systematic uncertainties, including bulk/surface selection and combined efficiencies, into account through the construction of the likelihood function. The aim of the analysis developed is to provide a reliable statistical forecast of positive signals.

The CDEX-10 detector array with a target mass of the range $10 \mathrm{~kg}$ has provided results on low-mass WIMP searches [27] and will be installed in a new $1700 \mathrm{~m}^{3}$ large $\mathrm{LN}_{2}$ at CJPL-II [25]. In the meantime, the homemade germanium detectors with ultralow-background electronics are being pursued, which establishes a platform to study the crucial technologies and foresees to suppress the background.

\section{ACKNOWLEDGMENTS}

This work is supported by the National Key Research and Development Program of China (Grant No. 2017YFA0402200), the National Natural Science Foundation of China (Grants No. 11505101, No. 11725522, No. 11675088, No. 11475099, and No. 11475092), the Fundamental Research Funds for the Central Universities (Grant No. 20822041C4030), and Tsinghua University Initiative Scientific Research Program (Grant No. 20197050007). The authors of affiliations 5 and 11 participated as members of TEXONO Collaboration.
[1] R. D. Peccei and H. R. Quinn, Phys. Rev. D 16, 1791 (1977).

[2] S. Weinberg, Phys. Rev. Lett. 40, 223 (1978).

[3] F. Wilczek, Phys. Rev. Lett. 40, 279 (1978).

[4] J. E. Kim, Phys. Rev. Lett. 43, 103 (1979).
[5] M. Shifman, A. Vainshtein, and V. Zakharov, Nucl. Phys. B166, 493 (1980).

[6] M. Dine, W. Fischler, and M. Srednicki, Phys. Lett. 104B, 199 (1981). 
[7] A. R. Zhitniskiy, Yad. Fiz. 31, 497 (1980).

[8] P. Gondolo and G. G. Raffelt, Phys. Rev. D 79, 107301 (2009).

[9] Z. Ahmed et al. (CDMS Collaboration), Phys. Rev. Lett. 103, 141802 (2009).

[10] C. E. Aalseth et al. (CoGeNT Collaboration), Phys. Rev. Lett. 106, 131301 (2011).

[11] N. Viaux, M. Catelan, P. B. Stetson, G. G. Raffelt, J. Redondo, A. A. R. Valcarce, and A. Weiss, Phys. Rev. Lett. 111, 231301 (2013).

[12] E. Armengaud et al. (EDELWEISS Collaboration), J. Cosmol. Astropart. Phys. 11 (2013) 067.

[13] H. An, M. Pospelov, J. Pradler, and A. Ritz, Phys. Lett. B 747, 331 (2015).

[14] N. Abgrall et al. (Majorana Collaboration), Phys. Rev. Lett. 118, 161801 (2017).

[15] D. S. Akerib et al. (LUX Collaboration), Phys. Rev. Lett. 118, 261301 (2017).

[16] C. Fu et al. (PandaX-II Collaboration), Phys. Rev. Lett. 119, 181806 (2017).

[17] E. Aprile et al. (XENON Collaboration), Phys. Rev. D 95, 029904 (2017).

[18] S. K. Liu et al. (CDEX Collaboration), Phys. Rev. D 95, 052006 (2017).

[19] E. Aprile et al. (XENON Collaboration), Phys. Rev. D 96, 122002 (2017).

[20] E. Armengaud et al. (EDELWEISS Collaboration), Phys. Rev. D 98, 082004 (2018).

[21] M. K. Singh, L. Singh, M. Agartioglu, V. Sharma, V. Singh, and H. T. Wong, Chin. J. Phys. 58, 63 (2019).

[22] E. Aprile et al. (XENON Collaboration), Phys. Rev. Lett. 123, 251801 (2019).

[23] P. Adhikari et al., Astropart. Phys. 114, 101 (2020).

[24] K. J. Kang et al., Front. Phys. 8, 412 (2013).

[25] J. P. Cheng et al., Annu. Rev. Nucl. Part. Sci. 67, 231 (2017).

[26] Q. Yue et al. (CDEX Collaboration), Phys. Rev. D 90, 091701 (2014).
[27] H. Jiang et al. (CDEX Collaboration), Phys. Rev. Lett. 120, 241301 (2018).

[28] L. Wang et al. (CDEX Collaboration), Sci. Chin. Phys. Mech. Astron. 60, 071011 (2017).

[29] Z. Z. Liu et al. (CDEX Collaboration), Phys. Rev. Lett. 123, 161301 (2019).

[30] L. T. Yang et al. (CDEX Collaboration), Phys. Rev. Lett. 123, 221301 (2019).

[31] Y. Wu et al., Chin. Phys. C 37, 086001 (2013).

[32] W. Zhao et al. (CDEX Collaboration), Phys. Rev. D 88, 052004 (2013).

[33] W. Zhao et al. (CDEX Collaboration), Phys. Rev. D 93, 092003 (2016).

[34] L. T. Yang et al. (CDEX Collaboration), Chin. Phys. C 42, 023002 (2018).

[35] J. L. Ma et al., Appl. Radiat. Isot. 127, 130 (2017).

[36] D. B. Kaplan, Nucl. Phys. B260, 215 (1985).

[37] M. Srednicki, Nucl. Phys. B260, 689 (1985).

[38] S. Andriamonje et al., J. Cosmol. Astropart. Phys. 12 (2009) 002.

[39] J. Redondo, J. Cosmol. Astropart. Phys. 12 (2013) 008.

[40] A. M. Green, Mod. Phys. Lett. A 27, 1230004 (2012).

[41] F. Alessandria et al., J. Cosmol. Astropart. Phys. 05 (2013) 007.

[42] A. Derevianko, V. A. Dzuba, V. V. Flambaum, and M. Pospelov, Phys. Rev. D 82, 065006 (2010).

[43] M. Pospelov, A. Ritz, and M. Voloshin, Phys. Rev. D 78, 115012 (2008).

[44] L. T. Yang et al., Nucl. Instrum. Methods Phys. Res., Sect. A 886, 13 (2018).

[45] J. N. Bahcall, Phys. Rev. 132, 362 (1963).

[46] W. Bambynek et al., Orbital electron capture by the nucleus, Technical Report, 1976.

[47] G. Cowan, K. Cranmer, E. Gross, and O. Vitells, Eur. Phys. J. C 71, 1554 (2011).

[48] A. L. Read, J. Phys. G 28, 2693 (2002). 